\title{
Ireo Karazana Zavamaniry Tena Fampiasa Amin'Ny Toerana Iray
}

\author{
Fidy Ratovoson, Henry Jaona, Justin Rabemananjara, \\ Juliette Marovavy, Clara Antonia, Marie Jeanne, \\ Clara Aimé Raholiarivelo, Emilien Razaniry, Ignace \\ Robena, Liva, Rogin, Delphin Razanajatovo, Esisy \\ Fanomezantsoa, Justin Rabezanahary, Velomary, Nirina
}

\section{Fampidirana}

Nandritra ny herinandron'ny 21 martsa 2005 dia nototosaina tao amin'ny KNARP (Karoka Nasionaly Ampiharana ny Raki-Panafody) Antananarivo ny atrika'asa fianarana ny fifandraisan'ny koltora, ny olona, ny zavamaniry ary ny tontolo iainana. Ny atrik'asa dia nokarakarain'ny Missouri Botanical Garden (MBG) sy ny KNARP izay misolotena ny "International Cooperative Biodiversity Group" ao anatin'ny tetik'asa hikarohana fanafody vaovao eto Madagasikara.

Nizara roa moa ny fomba nitondrana an'ity atrik'asa ity ka ny tapany voalohany dia fampianarana an-dakilasy izay natao tao Antananarivo ary ny tapany faharoa fampiharana eny ambanivohitra ka tamin'ireo tanàna manodidina ny alan'Analalava ao anatin'ny kaominin'ny Mahavelona (Foulpointe) no voasafidy nanaovana izany.

Nisy lohahevitra maromaro nozaraina tamin'ny mpandray anjara hanaovana ny fampiharana. Ny fanadiahadiana izay nataoko dia mikasika Ny zavamaniry tena ilain'ny olona amin'ny fiainany andavanandro amin'ny toerana iray. Ity lahatsoratra ity izany dia mandravona ny valin'ny fanadihadiana natao tao Morarano mikasika an'io lohahevitra io.

I Morarano dia tanàna eo amin'ny 5 km atsimo andrefan'ny alan'Analalava (saritany 1), sahabo ho $15 \mathrm{~km}$ ao atsimo andrefan'i Mahavelona (renivohitry ny kaominina), ao anatin'ny faritanin'ny Toamasina. Ny maridrefy ara-jeografika misy azy dia ny latitida $17^{\circ} 43^{\prime} 54^{\prime \prime} \mathrm{S}$ (Atsimo) sy ny longitida $49^{\circ} 25^{\prime} 38^{\prime \prime} \mathrm{E}$ (Atsinana). Ny haavony raha oharina amin'ny ranomasina dia $70 \mathrm{~m}$ eo ho eo. Avy amin'ny foko Betsimisaraka ny ankamaroan'ny mponina ao amin'ity tanàna ity ary mahatratra hatramin'ny telonjato (300) ny isany. Saika mpamboly avokoa izy ireo.

\section{Tanjona}

Ny tanjon'ny fanadihadiana izay natao dia mamantatra ny zavamaniry izay tena akaiky ny olona ao an-tanàna, tarafana avy amin'ny fampiasany azy an-davanandro.

\section{Fomba Nanaovana Ny Fanadiahadiana}

Talohan'ny fanadihadiana dia nisy ny fihaonana tamin'ny tangalamena sy ny olom-boafidy tompon'andraikitra ao amin'ny tanàna, niaraka tamin'ny solotenam-pokonolona. Nohazavaina tamin'izany ny anton-dia ary nanotaniana ny hevitr'izy ireo ny amin'ny fanadihadiana izay hatao. Taorian'ny resaka nifanaovana moa dia nanaiky izy ireo fa tsy misy ny olana amin'ny fiarahana miasa aminay.

Talohan'ny nanombohana ny asa koa dia napetraka ny tsangan-kevitra hoe rehefa hanotaniana ny olona iray momba ny fiainany sy ny zavatra hampiasainy dia izay mandalo matetika eo amin'ny masony sy/na nampiasaina vao haingana no tadidiny. Noho izany dia fanadihadiana mandeha tsirairay tamin'ny olona maromaro no na-

\section{Correspondence}

Fidy Ratovoson, Missouri Botanical Garden, BP 3391, Antananarivo 101, MADAGASIKARA.

fidy.ratovoson@mobot-mg.org

Henry Jaona, Justin Rabemananjara, Juliette Marovavy, Clara Antonia, Marie Jeanne, Clara Aimé Raholiarivelo, Emilien Razaniry, Ignace Robena, Liva, Rogin, Delphin Razanajatovo, Esisy Fanomezantsoa, Justin Rabezanahary, Velomary, Nirina, Morarano, Fokontany Morarano, Kaominina Mahavelona , Toamasina, MADAGASIKARA.

Ethnobotany Research \& Applications 3:329-335 (2005) 


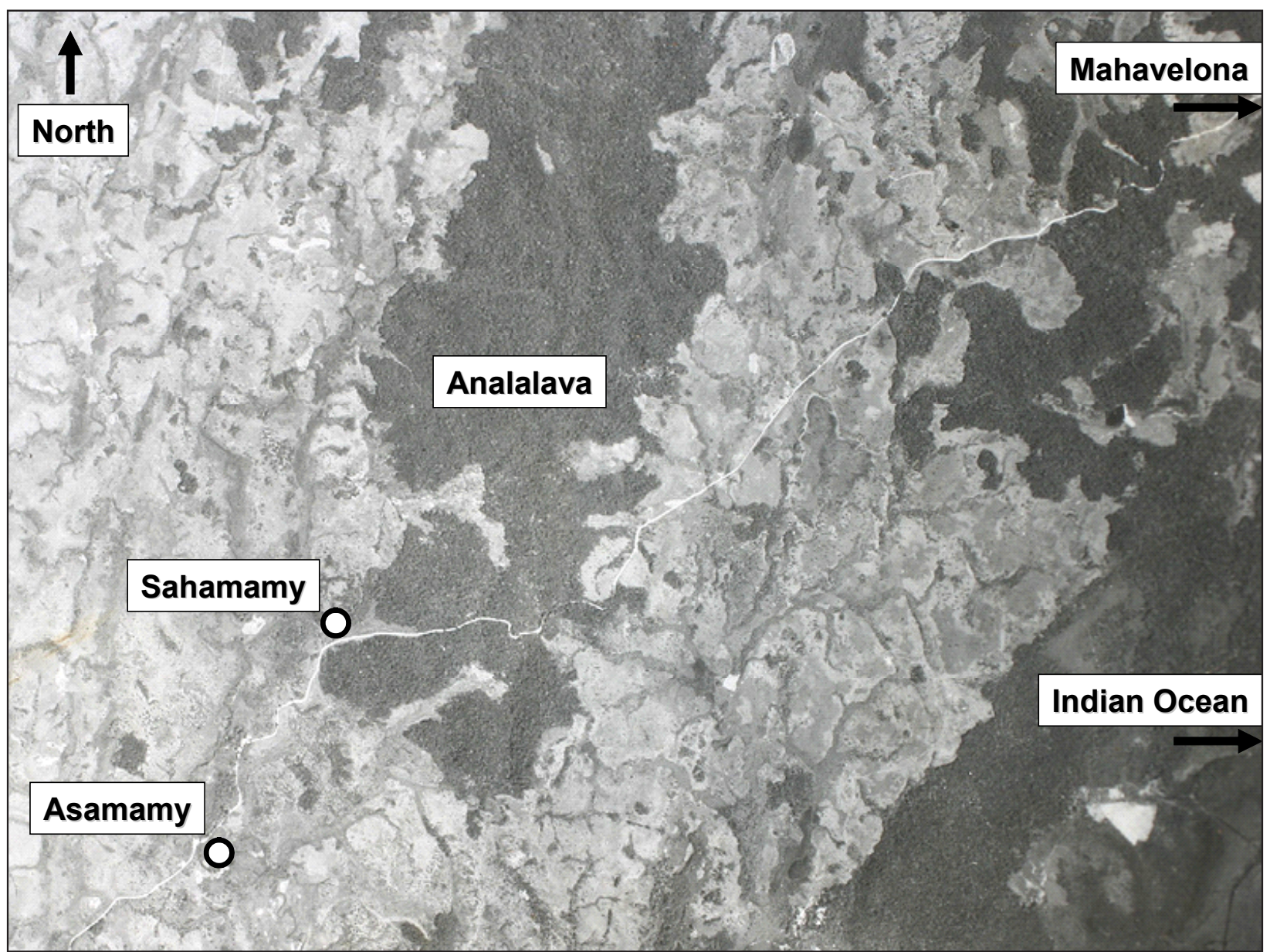

Saritany 1. Analalava.

tao. Nohezahana ho tafiditra tao ny fananahana samihafa (lahy, vavy) ary koa ny sokajin-taona nisy (zaza, tanora, lehibe). Ireto ny fanotaniana napetraka tamin'izany:

1. Afaka miteny ny zavamaniry fampiasanao amin'ny fiainana andavanandro ve ianao?

2. Inona no fampiasana azy?

3. Aiza ianao no maka azy?

4. Toy ny inona ny habetsany ilainao ary isaky ny inona ianao no maka azy?

5. Betsaka ve sa vitsy io zavamaniry io?

6. Raha vitsy dia inona no mety hasolo azy raha sanatria ka tsy hita intsony izy?

\section{Vokatra}

Olona dimy ambifolo (15) no nanaovana fanadihadiana (Tabilao 1). Fito amin'izy ireo no lehilahy ary 8 vehivavy. Latsaky ny 15 taona ny telo tamin'izy ireo, eo anelanelan'ny $15-30$ taona ny 6 ary mihoatra ny 30 taona ny 5 . Samy mponina ao Morarano daholo izy ireo sady

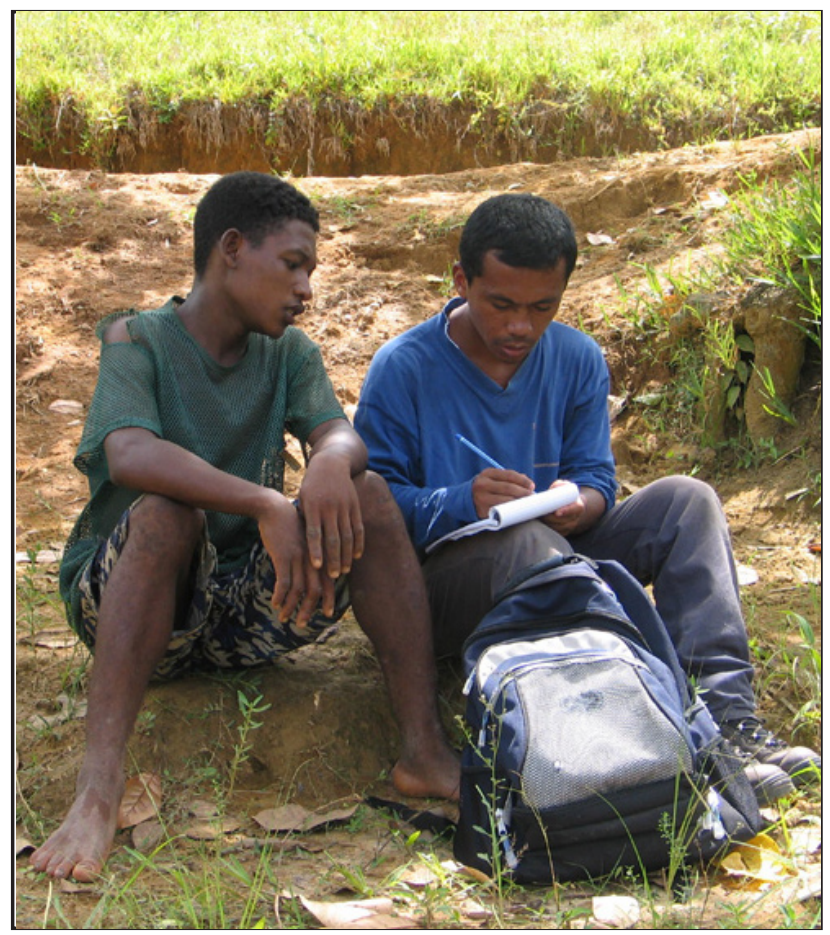




\section{Ratovoson et al. - Ireo Karazana Zavamaniry Tena Fampiasa Amin'Ny Toerana Iray}

Tabilao 2. Ireo hazo fampiasa araka ny sokajy hampiasana azy.

\begin{tabular}{|c|c|c|c|c|c|}
\hline & $\begin{array}{c}\text { Hanaovana } \\
\text { trano }\end{array}$ & Atao sakafo & $\begin{array}{c}\text { Hanaovana } \\
\text { kilalao }\end{array}$ & Kitay & $\begin{array}{c}\text { Hafa (haingo, fanafody, } \\
\text { hahazoana vola) }\end{array}$ \\
\hline Akondro & & $\mathrm{X}$ & & & \\
\hline Ampalibe & $x$ & $x$ & & & \\
\hline Andrarezina & $\mathrm{X}$ & & & & \\
\hline Atafana & & & & & $\mathrm{X}$ \\
\hline Belle de jour & & & & & $x$ \\
\hline Bonara & $X$ & & & & \\
\hline Canelle & & & & & $x$ \\
\hline Dindemo & $\mathrm{X}$ & & & & \\
\hline Fary & & $\mathrm{X}$ & & & \\
\hline Fotatra & $x$ & & $x$ & & \\
\hline Gavo & $\mathrm{X}$ & & & $X$ & \\
\hline Gavosina & & & & & $\mathrm{X}$ \\
\hline Grevillia & $\mathrm{X}$ & & $\mathrm{X}$ & & \\
\hline Harongana & $x$ & & & $X$ & $x$ \\
\hline Hasina & & & & & $\mathrm{X}$ \\
\hline Hazoambo & & & & & $x$ \\
\hline Kafe & & & & & $\mathrm{X}$ \\
\hline Kininina & $x$ & $X$ & $X$ & $x$ & \\
\hline Labiza & & & & & $x$ \\
\hline Lingosa & & & & $x$ & \\
\hline Litchi & & $x$ & & & \\
\hline Makaranana & & & & $X$ & \\
\hline Mananasy & & $X$ & & & \\
\hline Manga & & $X$ & & & \\
\hline Mangahazo & & $x$ & & & \\
\hline Mongy & & & & $x$ & \\
\hline Monkaranana & $\mathrm{X}$ & & & & \\
\hline Ovy & & $x$ & & & \\
\hline Palmier & & & $x$ & & \\
\hline Ranominty & $\mathrm{X}$ & & & $X$ & \\
\hline Ravinala & $x$ & & $x$ & & \\
\hline Saonjo & & $X$ & & & \\
\hline Soanambo & & $x$ & & & \\
\hline Tendrofono & $x$ & & & & \\
\hline Tsomanga & & $x$ & & & \\
\hline Vahizato & $X$ & & & & \\
\hline Varo & $x$ & & & & $x$ \\
\hline Vary & & $x$ & & & \\
\hline Voangy Ala & & $x$ & & & \\
\hline Voangy Antanàna & & $x$ & & & \\
\hline
\end{tabular}




\begin{tabular}{|l|c|c|c|c|c|}
\hline & $\begin{array}{c}\text { Hanaovana } \\
\text { trano }\end{array}$ & Atao sakafo & $\begin{array}{c}\text { Hanaovana } \\
\text { kilalao }\end{array}$ & Kitay & $\begin{array}{c}\text { Hafa (haingo, fanafody, } \\
\text { hahazoana vola) }\end{array}$ \\
\hline Voanio & & $\mathrm{X}$ & & & $\mathrm{X}$ \\
\hline Voapaka & $\mathrm{X}$ & & & & \\
\hline Volo & $\mathrm{X}$ & & $\mathrm{X}$ & & \\
\hline Zatsy & & $\mathrm{X}$ & & & \\
\hline Zavoka & & $\mathrm{X}$ & & & \\
\hline
\end{tabular}

lehibe tao, afa-tsy vehivavy iray 34 taona izay vao 9 taona no nipetraka tao.

Nisy karazany dimapolo (50) ny zavamaniry fantatra taorian'ny fanadiahadiana fa tena hampiasain'ny olona amin'ny fiainany Andavanandro. Ireto avy izy ireo manaraka ny habetsaky ny olona nisafidy azy : Ampalibe, Kininina, Mangahazo, Vary, Akondro, Fary, Litchi, Ranominty, Harongana, Ravinala, Voanio, Gavo, Voangy Antanàna, Fotatra, Grevillia, Hasina, Hazoambo, Kafe, Sakoana, Soanambo, Tsomanga, Varo, Volo, Zavoka, Andrarezina, Antsotry, Apemba, Atafana, Belle de jour, Bonara, Canelle, Dindemo, Gavosina, Labiza, Legioma, Lingosa, Monkaranana, Mananasy, Manga, Mongy, Ovy, Palmier,
Saonjo, Tendrofono, Vahizato, Voangy Ala, Voangy Dia, Voapaka, Zatsy.

Tabilao 1. Famitinana ireo olona nanaovana fanadihadiana.

\begin{tabular}{|l|c|c|c|}
\hline Taona & Lehilahy & Vehivavy & Totaliny \\
\hline Latasaky ny 15 taona & 2 & 1 & 3 \\
\hline $15-30$ taona & 5 & 2 & 7 \\
\hline Mihoatra ny 30 taona & 2 & 3 & 5 \\
\hline Totaliny & $\mathbf{9}$ & $\mathbf{6}$ & 15 \\
\hline
\end{tabular}

Ny tabilao faha-2 dia mampiseho ny fizarazaran' ireo zavamaniry araka ny fomba fampiasana azy. Fito ambinifolo

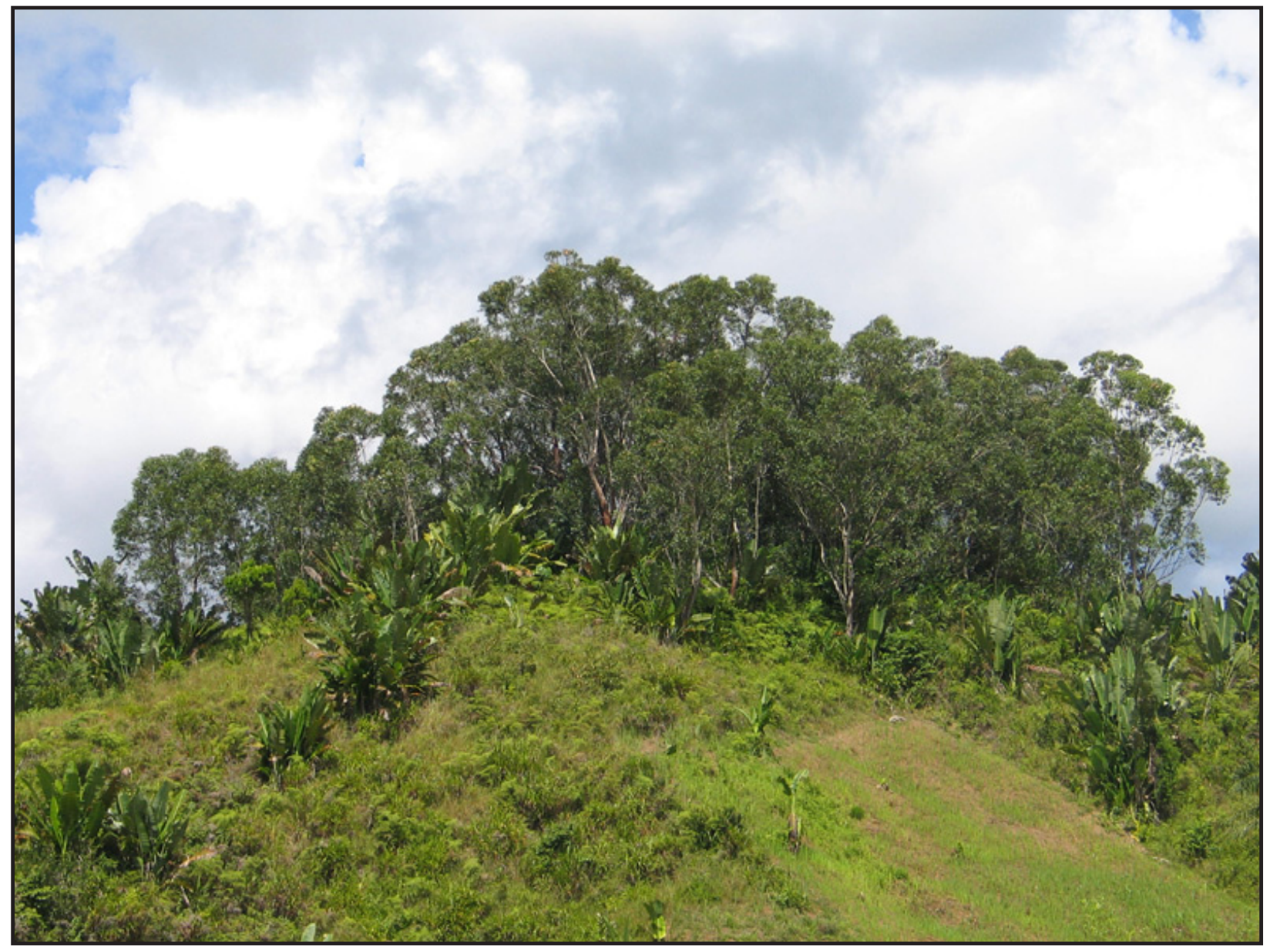



Toerana Iray

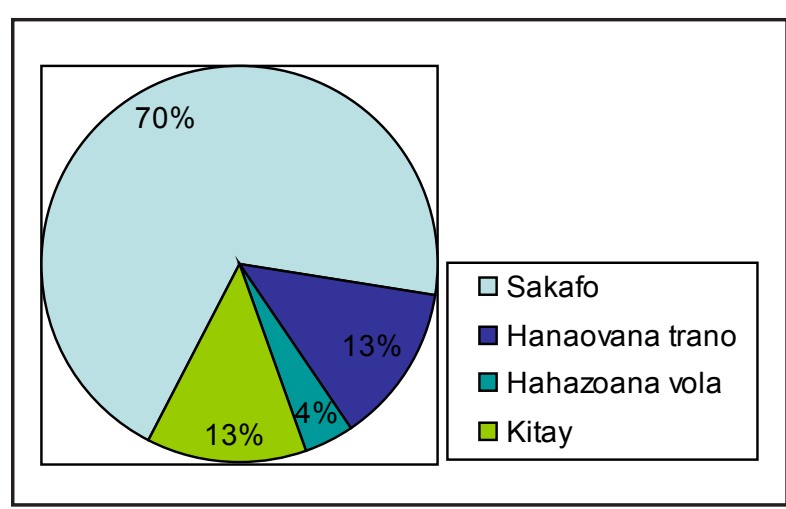

Kisary 1. Habetsaky ny zavamaniry fampiasan'ny vehivavy arakaraky ny fomba hampiasany azy.

(17) karazana na $34 \%$ dia fanaovana trano, sivy ambifolo (19) karazana na $38 \%$ fanaovana sakafo, 7 karazana na $14 \%$ fanaovana kilalao, 6 karazana na $12 \%$ fanaovana kitay, ary iraika ambifolo (11) karazana na $22 \%$ fanaovanjavatra hafa toy ny fanafody, haingo, fefy sns...

Ireto avy ireo hazo tena betsaka mpampiasa (i.e. arakaraky ny habetsaky ny olona nisafidy azy izay ao anaty fonosana): Ampalibe (8), Kininina (8), Mangahazo (7), Vary (7), Akondro (6), Fary (6), Litchi (5), Ranominty (5), Harongana (5), Ravinala (4), Voanio (4), Voangy Antanàna (4), Fotatra (4), Grevillia (4), Hasina (4), Hazoambo (4), Kafe (3), Sakoana (3), Soanambo (3), Tsomanga (2), Varo (2), Volo (2), Zavoka (olona 2).

Dimy ambiroapolo (25) ny karazan-javamaniry nosafidian'ny vehivavy. Ny efatra ambiroapolo (24) na 96\% amin'ireo dia ilainy manodidina ny tokatrano (hanaovana sakafo, atao kitay, hanamboarana trano) (Kisary 1). Iraika amby efapolo (41) ny karazan-javamaniry nosafidian'ny lehilahy (Kisary 2). Hita fa saika mahasahana ny akolafinjavatra rehetra ny lehilahy. Voaporofo ilay fiteny Malagasy hoe ny lehilahy ny lohan'ny fianakaviana.

Fanamarihana: Taorian'ny tatitra natao tamin'ny vahoaka no nanamarihan'izy ireo fa maka hazo any Analalava ihany izy ireo rehefa mila hazo matoy hanaovana trano saingy mila maka fahazahoan-dalana manokana avy any amin'i lehiben'ny ala izy ireo amin'izany. Noho io antony farany io dia malaina ny olona mandeha any.

Ny dimapolo isan-jaton'ny zavamaniry rehetra (50\%) hampiasain'ny olona dia manimba tanteraka azy, ary ny dimapolo isan-jato (50\%) hafa dia ampahany ihany no alainy ka tsy manimba azy ireo. Mikasika ny toerana hangalan'ny olona ny zavamaniry hampiasainy indray dia mizara telo mazava tsara (Kisary 3). Voalohany, ireo alaina mivantana eny an-tsaha (anaty savoka sy ala). Mahatratra hatrany amin'ny $58 \%$ izany. Faharoa, azo avy amin'ny fambolena eny an-tsaha (lavitry ny tanàna) izay midadasika. Ny hazo fanaovan-trano (kininina), ny vary

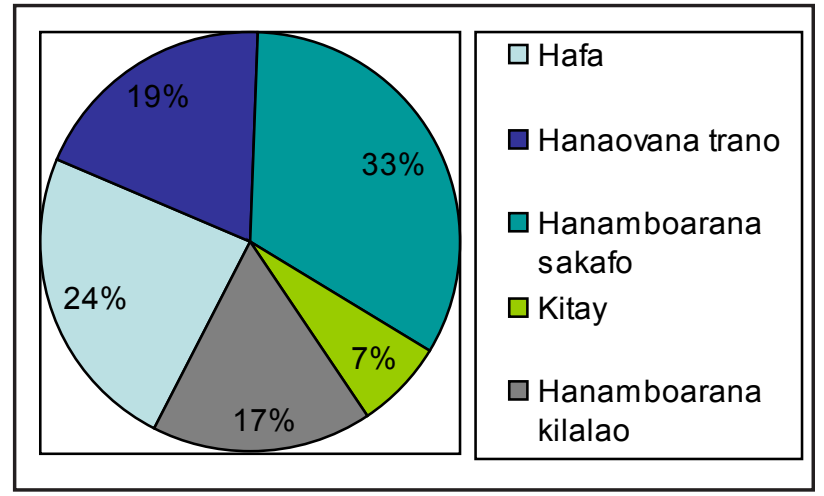

Kisary 2. Habetsaky ny zavamaniry fampiasan'ny lehilahy arakaraky ny fomba hampiasany azy.

ary ny mangahazo no betsaka amin'izany. Mahatratra hatrany amin'ny $22 \%$ io sokajy io. Farany dia tsy lavitra ny toerana honenany izany hoe manodidina ny trano. Tafiditra ao anatin'izany ny karazam-boankazo, legioma ary ny hazo hanaovana haingo.

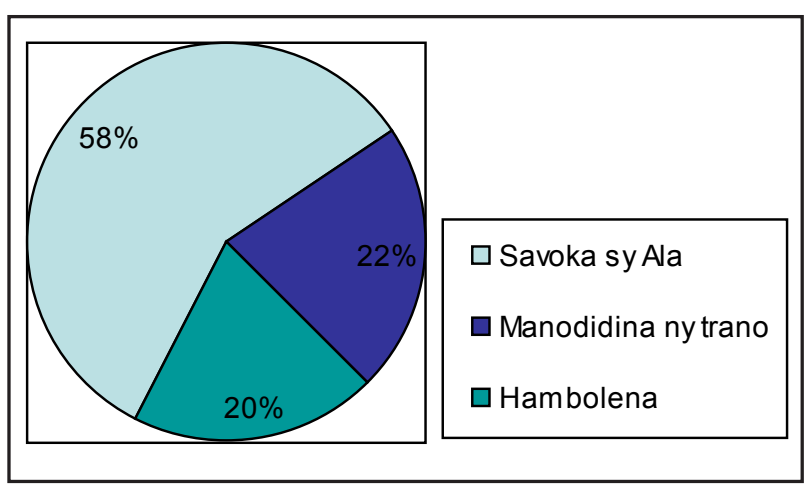

Kisary 3. Fizaran'ny toerana hangalan'ny olona ireo Zavamaniry.

\section{Famaranana}

Taorian'ny fanadihadiana dia misy tsoan-kevitra vitsivitsy azo ambara ireto :

1. Ny fiainana andavan'andro no tena hifontoran'ny olona. Noho izany, vitsy (olona 2) ny olona nieritreritra zavatra mba hahazoana vola.

2. Tsy misy hifandraisany firy loatra amin'ny alan'Analalava ny asa andavan'andron'ny mponina ao Morarano. Ny hazo hita amin'ny savoka sy ny hazo hambolena no tena betsaka hampiasaina.

3. Anisan'ny tsy hita tao anatin'ny fanadihadiana ny fampiasana ny zavamaniry ho fanafody. Tsy fantatra tato anatin'ity fanadihadiahana ity ny antony nahatonga izay ary mendrika hanaovana fanadihadiana manokana. 
4. Tsy halaim-panahy hampiasa zavatra tsy fahita firy na saro-tadiavina ny olona. Ny totolo manodinina azy sy izay akaiky azy no tena hampiasainy.

Farany, noho ny fahateren'ny fotoana dia voafetra ihany ny isan'ny olona afaka nanaovana fanadihadiana. Vokatr'izay dia maro ny fanotaniana manitikitika mety tsy ho voavalin'ity fanadiahadiana ity kanefa mety hilaina amin'ny fomba ho fiarovana ny alan'Analalava. Ohatra azo tsongaina amin'izany ny hoe misy “fiatraikany amin'ny fahasimban'ny alan'Analalava ve ny fahalaviran'ny tanàna iray ?" Ohatra hafa ny fanotaniana hoe "misy fivoarany ve ny fampiasana ny zavamaniry arakaraky ny sokajin-taona sy ny fananahana ?" Inoana fa mbola maro ny fikarohana hataon'ny tompon'andraikitra mikasika izay fifandraisan'ny koltora, ny olona, ny zavamaniry ary ny totolo iainana izay.

\section{Fisaorana}

Isaorana manokana ny mponina tao amin'ny Fonkotany Morarano, Foulpointe tamin'ny fandraisana sy ny fiarahana-miasa nandritra ny andro nanaovana ny fanadihadiana. Manolotra fisaorana betsaka ho an'Andriamatoa Will McClatchey sy Ramatoa Lisa Gollin tamin'ny fampianaran'izy ireo momba ny fifandraisan'ny koltora, ny olona, ny zavamaniry ary ny tontolo iainana. Isaorana ihany koa ny fitondram-panjakana Malagasy amin'ny alalan'ny departemantan'ny Rano sy ny Ala nanome alalana tamin'ny fanaovana ny atrik'asa. Farany, maneho fisaorana ny mpamatsy vola, dia ny National Institute of Health tamin'ny alalan'ny tetik'asa International Cooperative Biodeversity Group (ICBG) tarihin'i Pr David Kingston.

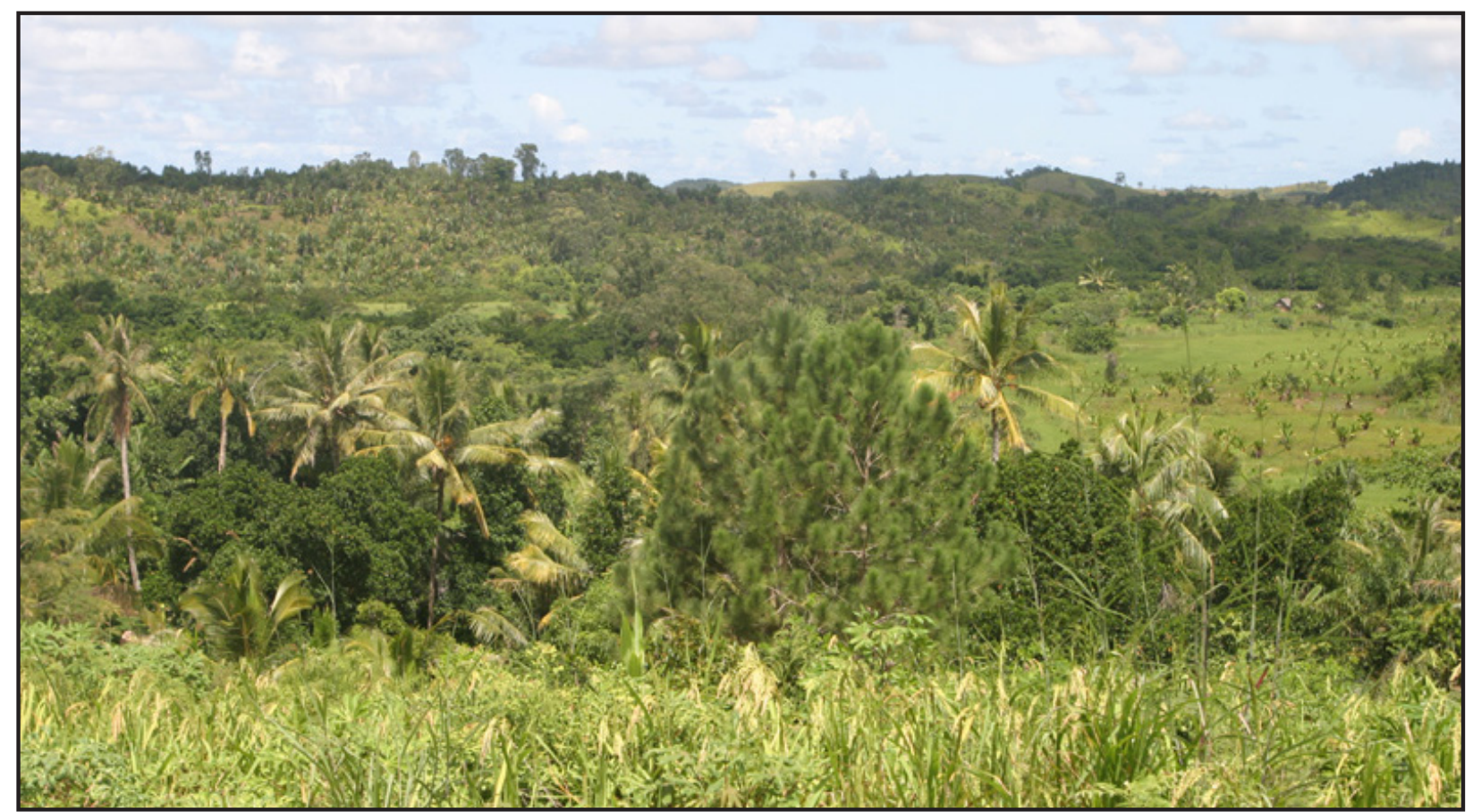

